\title{
Integrating Artificial Intelligence, Theory, Modeling and Experiments - Perspectives, Challenges, and Opportunities in Materials and Manufacturing
}

\author{
Guang $\operatorname{Lin}^{1, *}$ and $\mathrm{Na} \mathrm{Lu}{ }^{2, *}$ \\ Received: 10 September 2020; Accepted date: 21 September 2020. \\ Article type: Editorial article.
}

Artificial intelligence (AI)/machine learning (ML) are an active research field, which has shown a great success in various commercial applications. It will play an important role and have a great impact on many fields of science and engineering, in particular materials and manufacturing. In the past decades, AI and ML have become a crucial complement to theoretical, computational modeling, and experimental aspects in Engineering and Science. AI and ML models have great potentials particularly in the research areas where the mechanism is still not completely well-understood, or the computational models are too expensive to run to obtain accurate solutions at desired spatiotemporal resolutions. In this work, we introduce some recent work and promising research directions to integrate AI and ML in Engineering and Science. We attempt to provide a broader perspective, open challenges and unique opportunities on integrating AI, theory, modeling and experiments in the fields of materials and manufacturing.

First, experiments or computational models can be employed to generate data to train the AI/ML models. The trained $\mathrm{AI} / \mathrm{ML}$ models can be viewed as a fast surrogate model of the corresponding time-consuming experiments or computational models. A convolutional encoder-decoder networks with quantified uncertainty (ConvPDE-UQ) ${ }^{[1]}$ was developed to predict the solutions of partial differential equations on varied domains, which was much faster than the traditional finite element solver. A deep neural networks named Peri-Net ${ }^{[2]}$ was designed for analysis of crack patterns, which is much faster than the peridynamics solver. A deep neural process with a

\footnotetext{
${ }^{I}$ Department of Mathematics, School of Mechanical Engineering, Purdue University, West Lafayette, IN 47906, USA.

${ }^{2}$ Lyles School of Civil Engineering, School of Materials Engineering, Birck

Nanotechnology Center, Purdue University, West Lafayette, IN 47906, USA.

*E-mail: guanglin@purdue.edu (G. Lin), luna@purdue.edu (N. Lu).
}

quantified uncertainty capability named Peri-Net-Pro ${ }^{[3]}$ was developed for analysis of crack patterns.

Second, theory and physics laws can be integrated with $\mathrm{AI} / \mathrm{ML}$ models. The application of AI/ML models in science and engineering domains is facing some grand challenges due to the large data requirements and lack of generalizability. Recently various novel AI/ML models ${ }^{[4-8]}$ have been proposed to integrate both scientific knowledge and data together

Third, AI/ML models also can be employed to automatically discover theory, in particular, the physical laws. Various AI/ML models ${ }^{[9-1]}$ have been developed to automatically discover ordinary differential equations and partial differential equations. Uncertainty quantifications for the discovery of physical laws using AI/ML models have also been investigated. ${ }^{[9,10]}$

Fourth, in many applications in materials and manufacturing, it is expensive or time-consuming to collect experimental or simulation data. To resolve such challenges, active learning models can be employed to design optimal experiments and computational model simulations to greatly enhance the predictive capability of the AI/ML models with reduced experimental or simulation data size. An adaptive design criterion combining the D-optimality and the maximin space-filling criterion has been designed and demonstrated its capability.

Fifth, in materials and manufacturing, there are various multi-fidelity computational models and experiment instruments. How to integrate the data from all the models and experiment instruments is a grand challenge. Recently advanced multi-fidelity models ${ }^{[13]}$ have been developed to integrate multi-resolution data generated from multi-fidelity computational models or experiments for training and prediction. Advanced optimization algorithms ${ }^{[10,14-15]}$ have been developed for tuning the hyperparameters of the deep neural networks. 


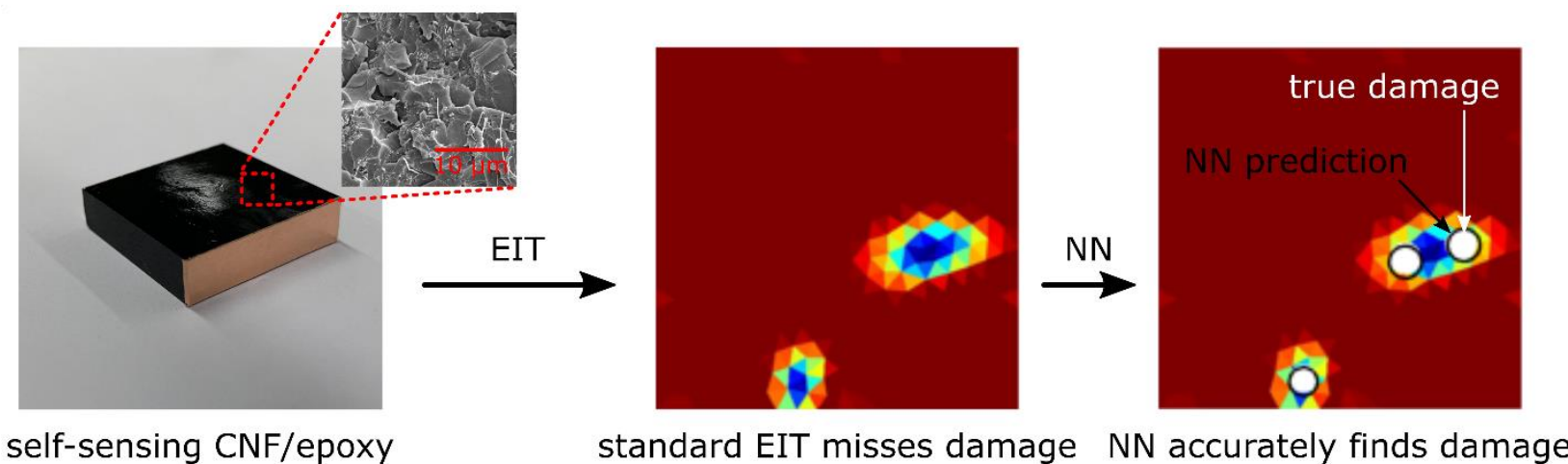

Fig. 1 Piezoresistive CNF/epoxy. Left: Post-manufactured measurement specimen with electrodes applied. Middle: standard EIT misses damage, Right: Neural Network accurately finds damage.

One of the critical challenging research questions in materials science is how to accurately discover the connection between materials and manufacturing and obtain a fundamental understanding of process-structure-property relation. Can we employ AI to help us build such a processstructure-property relation mapping, which in return will shed the light in designing unique materials behaviors through artificial-intelligence guided novel manufacturing process. Convolutional Neural Networks have been constructed to predict mechanical properties from microstructure images in fiber-reinforced polymers, A neural network-aided electrical Impedance tomography was developed for real-time precise damage characterization in self-sensing materials as shown in Fig. 1. A neural network ${ }^{[12]}$ was employed for infrared thermal imaging-based crack detection.

We expect AI/ML models will be further developed to address the challenges in materials and manufacturing and create a new paradigm, which will make use of domainspecific knowledge and focusing on exploring a deeper coupling of $\mathrm{AI} / \mathrm{ML}$ methods with scientific knowledge and physical laws. It will greatly speedup the designing process of the next-generation materials and their related manufacturing studies.

\section{Acknowledgements}

G. Lin gratefully acknowledge the support from the National Science Foundation (DMS-1555072, DMS-1736364, CMMI1634832, and CMMI-1560834), Brookhaven National Laboratory Subcontract 382247, ARO/MURI grant W911NF15-1-0562 and Department of Energy DE-SC0021142.

\section{Supporting information}

Not applicable

\section{Conflict of Interest}

There are no conflicts to declare.

\section{References}

[1] N. D. Winovich, K. Ramani, G. Lin, J. Comput. Phys., 2019, 394, 263-279, doi: 10.1016/j.jcp.2019.05.026.

[2] M. Kim, N. Winovich, G. Lin, W. Jeong, J. Peridy. Nonloc. Model., 2019, 1, 131-142 doi: 10.1007/s42102-019-00013-x.
[3] M. Kim, G. Lin, arXiv., 2020, 2005, 13461. https://arxiv.org/abs/2005.13461

[4] M. Alber, A. B. Tepole, W. R. Cannon, S. De, S. DuraBernal, K. Garikipati, G. Karniadakis, W. W. Lytton, P. Perdikaris, L. Petzold and E. Kuhl , NPJ. Digital Medicine, 2019, 2, 1-11, doi: 10.1038/s41746-019-0193-y.

[5] N. Baker, F. Alexander, T. Bremer, A. Hagberg, Y. Kevrekidis, H. Najm, M. Parashar, A. Patra, J. Sethian, S. Wild, K. Willcox, S. Lee, Technical Report. USDOE Office of Science, 2019, https://doi.org/ 10.2172/1478744.

[6] A. Karpatne, G. Atluri, J.H. Faghmous, M. Steinbach, A. Banerjee, A. Ganguly, S. Shekhar, N. Samatova, and V. Kumar, IEEE Tkde 2017, 29, 2318-2331, https://doi.org/ 10.1109/TKDE.2017.2720168.

[7] R. Rai and C. K. Sahu, IEEE Access 2020, 8, 71050-71073, https://doi.org/10.1109/ACCESS.2020.2987324.

[8] M. Raissi, P. Perdikaris, G.E. Karniadakis, J. Comput. Phys., 2019, 378, 686-707, https://doi.org/10.1016/j.jcp.2018.10.045.

[9] S. Zhang, G. Lin, Proc. Roy. Soc. London. Series A, 2018,

474, 20180305, https://doi.org/10.1098/rspa.2018.0305.

[10] Y. Wang, G. Lin, J. Comput. Phys., 2020, 401, 108969, https://doi.org/10.1016/j.jcp.2019.108968.

[11] S. L. Brunton, J. L. Proctor, J. N. Kutz, Proc. National Acade. Sci. U. S. A., 2016, 113, 3932-3937, doi: 10.1073/pnas. 1517384113.

[12] J. Yang, W. Wang, G. Lin, Q. Li, Y. Sun, Y. Sun, IEEE Access 2019, 7, 182060-182077, doi:

10.1109/ACCESS.2019.2958264.

[13] N. Ou, L. Jiang, G. Lin, Int. J. Numer. Meth. Eng., 2019, 119, 941-963, doi: 10.1002/nme.6079.

[14] W. Deng, Q. Feng, L. Gao, F. Liang, G. Lin, Inter. Conference on Machine Learning (ICML), 2020, 2008(V2), 05367, https://arxiv.org/abs/2008.05367.

[15] W. Deng, X. Zhang, F. Liang, G. Lin, Adv. Neural Inf. Pro. Sys.. 2019, 5563.

https://papers.nips.cc/paper/8794-an-adaptive-empiricalbayesian-method-for-sparse-deep-learning

Publisher's Note: Engineered Science Publisher remains neutral with regards to jurisdictional claims in published maps and institutional affiliations. 